\title{
De la problématique à la problématisation
}

$\mathrm{L}$

es phénomènes que nous traitons relèvent rarement de situations épurées dont la solution est techniquement évidente. Ils constituent souvent des questions mal structurées, des problèmes irréductibles, des sujets de controverses dont les solutions souvent divergentes ne se révèlent efficientes que dans le contexte et la temporalité où elles sont produites. Nous avons été conditionnés par nos habitudes de pensée à tirer des conclusions privilégiant des relations de cause à effet simples et linéaires. Or, la caractéristique des problèmes épineux (Rittel et Webber, 1973) est qu'ils ne peuvent pas être réduits à la résolution d'une cause unique. Ainsi, notre société, habituée à résoudre les problèmes en les décomposant et en les simplifiant comme cela est enseigné dans les écoles, comprend de moins en moins le monde dont elle fait pourtant partie. Les récents bouleversements liés à la crise sanitaire invitent, comme d'autres disciplines l'ont fait, à nous positionner par rapport à l'atteinte d'objectifs classiquement conceptualisés comme incompatibles voire antagonistes. Plus que jamais, la nécessité s'est faite jour d'une pensée propre à affronter les problèmes que les complexités posent aux chercheurs et aux managers emportés dans les méandres de phénomènes qui échappent à la raison. 
Ce dossier, créé à l'occasion du centenaire d'Edgar Morin, incite à se concentrer sur ces alternatives pour saisir les aspects observables des phénomènes comme manifestation de la discontinuité, de la non-linéarité, de l'incertitude, i.e. la multiplicité des phénomènes que l'on exprime sous le terme « complexité ». En effet, la « complexité » est devenue un terme passe-partout trop souvent perçu comme une dégradation de l'environnement par rapport à la capacité humaine à comprendre et à agir. Or, la complexité se contente de se révéler à nous sous bien des formes et des niveaux et n'invite pas à «botter en touche » ou au renoncement dans un monde où l'individu a de plus en plus de mal à en comprendre les dysfonctionnements et changements. Elle n'appelle pas non plus à une quête infinie et insatiable visant à découvrir les enchevêtrements de causes et de conséquences qui permettraient d'expliquer pourquoi un quelconque battement d'aile de papillon a pu être à l'origine d'une tempête. " À supposer que nous voudrions une observation exhaustive sur un objet, nous serions entraînés dans une spirale infinie des interactions auxquelles participe cet objet et dont il procède (...) La connaissance portée à l'absolue est autodestructrice. » (Morin, 1977, p. 355-356).

En fait, la complexité révèle les limites de la raison logique classique. Les manières avec lesquelles la recherche reste opérée de nos jours demeure, pour une large part, un héritage des dogmes cartésiens tendant à réduire l'objet de recherche en l'isolant de son sujet tout en délimitant son périmètre d'analyse. Recherchant surtout des causalités et des régularités, ces approches peinent à donner du sens à des cas d'instabilité, d'ambiguïté, de contingence ou de bifurcations, alors que les praticiens sont en permanence confrontés à ces situations. Or, la complexité du monde réel ne peut plus être perçue et représentée par un seul ensemble de règles déterministes, algorithmiques, ou encore par des mécanismes non déterministes et distribués mais occultant l'intuition, le flair, le rêve, l'imaginaire et l'émotion. En même temps, la pensée complexe ne vise pas, comme cela est souvent perçu, à supplanter les approches positivistes mais à les interroger aux endroits où elles cessent d'être opérationnelles (Morin et Le Moigne, 1999, p. 150). Il ne s'agit pas de deux manières interchangeables, mais de deux niveaux épistémiques complémentaires, correspondant à différentes manières de penser et d'étudier les phénomènes organisationnels.

La pensée complexe selon Edgar Morin (Morin, 1977, 1980, 1986, 1991, 2001, 2004) invite à repenser nos modes de réflexion. Elle ne prétend pas permettre de connaître un phénomène dans son intégralité (c'est-à-dire de manière exhaustive), mais à le comprendre dans son intégrité, c'est-à-dire par rapport à sa propre dynamique autogénérative en lien avec son environnement. Inspirée de la théorie des systèmes, de la cybernétique, de la théorie de l'information de Shannon, et de la systémique, la pensée complexe de Morin propose d'étudier un système conjointement dans sa globalité et dans le détail de ses composants. Le postulat de base est que l'on ne peut pas connaître le système sans en connaître les composants, de même qu'il est impossible de connaître les composants sans connaître le système dont ils font partie. L'étude d'une des parties de ce phénomène, ne peut alors se faire qu'à la lumière de ses interdépendances avec les autres parties et avec le tout. L'enjeu n'est donc pas d'identifier l'influence marginale de 
« facteurs isolés », mais la manière dont ils se combinent mutuellement, s'auto-alimentent, tout en montrant comment ils sont euxmêmes influencés par le système auquel ils contribuent, de même que par l'environnement avec lequel ils sont en interdépendance. Dans le domaine du management et de l'organisation, une réflexion sur la pensée complexe a été, entre autres, développée depuis près de 30 ans par les membres du réseau "Modélisation de la Complexité » (MCX, fondé par Jean-Louis Le Moigne) ${ }^{1}$. Une revue systématique des journaux internationaux classés en gestion menée en 2018 a révélé 8272 citations des travaux de Morin ${ }^{2}$. Pour sa part, la RFG a publié un numéro spécial en 2012 intitulé «Le management face à la complexité ». La complexité était donc vue comme un contexte face auquel le management se devait de s'adapter ou d'improviser. Comme l'écrivait Benoît Journé dans l'éditorial : « Nous avons choisi de ne pas définir de thématique générale a priori mais plutôt de la laisser prendre forme progressivement, en respectant au maximum la singularité et l'originalité de chaque contribution. Le thème de la complexité a donc émergé des discussions ouvertes par les articles retenus. » Bien qu'ils n'utilisaient pas la pensée complexe comme prisme conceptuel, les articles ont témoigné de cas d'études qui lui faisaient écho.

L'objectif du présent dossier n'était pas de chercher, à nouveau, à débattre sur des querelles épistémiques clivantes alors que le contexte que nous venons de vivre a rebattu les cartes des croyances et certitudes à partir desquelles la science s'est développée depuis le $\mathrm{XIX}^{\mathrm{e}}$ siècle. Ce dossier publie des articles qui ont contribué à une opérationnalisation de la pensée complexe dans la recherche sur les organisations en la considérant comme une approche alternative critique pour appréhender les phénomènes étudiés.

Les sciences n'ont jamais connu un tel essor, pourtant les réponses acquises se transforment bien vite en questions nouvelles qui posent, à leur tour, de nouveaux problèmes qui interrogent nos certitudes. De leur côté, les sciences de gestion, depuis qu'elles existent, se sont essentiellement attachées à proposer des dispositifs managériaux dont la légitimité résidait dans leur objectif à résoudre les problématiques des managers. Mêmes celles qui ont été anoblies du statut de best practices ont montré leurs limites lorsqu'il s'agissait de manager dans la complexité. L'objectif de ce dossier spécial de la RFG n'est donc pas tant de prétendre fournir des solutions ou des méthodologies qui pourraient être entendues comme des programmes de résolution de problèmes. Il s'inscrit dans l'aide aux managers à penser et modéliser les problèmes afin de les accompagner hors du réflexe simplificateur par lequel les problématiques sont souvent traitées en vue de rapidité à la résolution. Ainsi, la pierre angulaire que nous avons retenue réside moins dans la quête à la résolution du problème que dans la manière avec laquelle on se représente ce dernier au fil du temps. Le «sens du problème » constitue la « véritable marque de l'esprit scientifique »

1. Ce réseau et l'Association pour la pensée complexe, présidée par Edgar Morin, ont fusionné en 2000 pour former le réseau « Intelligence de la Complexité » (http://www.intelligence-complexite.org/nc/fr/ouverture/accueil.html).

2. Source : Bouiss (2018). 
(Bachelard) dès lors que le problème ne précède pas sa résolution (Martinet, 2006). Lorsque l'on étudie un phénomène complexe, la «problématisation » ne revient pas à poser de façon claire et nette le « bon problème » puisqu'il y a autant de façons de poser le problème qu'il $\mathrm{y}$ a de points de vue sur la question. Autant de façons qui, au demeurant, évoluent dès lors que l'on cherche à résoudre le problème, car le percevant alors sous de nouvelles contraintes et de nouveaux objectifs. Comme le précisait Simon (1995), le problème de conception est continuellement reformulé au cours du processus de conception.

L'enjeu d'une problématisation faisant sens avec la pensée complexe implique au chercheur d'adopter différents principes : - Ne pas réduire ou limiter l'objet de recherche à un cadre prédéterminé (ne serait-ce que par le domaine de spécialité du chercheur) mais, au contraire, le représenter par ses interrelations au sein de l'organisation dont il fait partie (principe hologrammatique) ainsi qu'au travers de sa dépendance à l'environnement (principe d'auto-éco-ré-organisation).

- Distinguer les composants du système étudié sans rejeter leur interdépendance (principe de conjonction versus de disjonction). En d'autres termes, il convient de ne pas réduire les liens à des relations de cause à effet mais de modéliser leur réciprocité et leur récursivité.

- Ne pas exclure des notions qui apparaissent comme opposées et faire sens avec des paradoxes et des contradictions pouvant être vus comme les deux faces d'une même pièce (principe dialogique). Par exemple, les notions d'ordre/désordre sont intrinsèques à la création et au fonctionnement des systèmes complexes et ce, qu'il s'agisse de systèmes naturels, biologiques ou sociaux, de la formation d'une étoile, des particules physiques ou des systèmes biologiques (Morin, 1977, p. 354). Un principe d'action managériale peut être d'instaurer des liens créateurs de valeur entre les pôles dialogiques identifiés (Avenier, 2019, p. 53).

- Faire sens de ce que fait le système par rapport aux finalités poursuivies pas à pas et non pas par une explication fonctionnelle (compréhension téléologique versus explication causale).

- Le principe de réintroduction du sujet. Tout objet ou processus artificiel contient les traces de son créateur, toute connaissance (théorique ou pratique) est une œuvre de l'esprit d'un être humain à un moment donné, dans un contexte donné et avec une intention donnée. Comme l'écrivait Lévi-Strauss (1950) « dans une science ou l'observateur est de même nature que son objet, l'observateur est lui-même une partie de son observation ». Selon la pensée complexe, il n'est donc pas suffisant de reconnaître la nonneutralité du chercheur observateur ou créateur. Outre le détail du contexte et du protocole scientifique, le chercheur doit faire sa propre anthropologie et dévoiler quels étaient ses croyances, intentions et états d'esprit liés à son projet.

Les articles retenus, au prix d'une très grande sélectivité, abordent différents cas par le prisme de la pensée complexe. Je remercie chaleureusement mes collègues Florence Rodhain, Jérémi Sauvage et Ousama Bouiss d'avoir joué avec moi le rôle de rédacteurs invités et sans lesquels ce projet n'aurait jamais pu être mené à son terme. Outre la motivation du réseau «Reliance en Complexité » d'une action symbolique à l'occasion du centenaire de 
notre ami Edgar Morin, nous pensions tout d'abord qu'un dossier spécial au sein de la communauté française portant sur la complexité allait drainer naturellement une quasi-totalité d'articles basés sur la pensée qui va avec. Or, sur les 24 soumissions, une grande majorité traitait de complexité ou de résolution de problèmes complexes sans inclure le changement épistémologique soutenu par la pensée morinienne. En d'autres termes, nous avions beaucoup de recherches qui traitaient de « complexité restreinte» et non de «complexité générale » (Morin, 2005). Aussi, dès le second tour, nous n'avons retenu que les articles dont nous étions convaincus qu'ils avaient le potentiel suffisant pour satisfaire l'objectif du dossier ainsi que les qualités académiques requises par la Revue française de gestion. Cette décision a eu pour conséquence de n'en sélectionner que quatre, au risque que le dossier ne puisse paraître si les auteurs n'étaient pas tous allés au terme du processus de révision demandé. Je tiens donc également à les remercier pour leur engagement et leur contribution.

La recherche conduite par Sandra Bertezene et David Valat nous offre le cas d'un projet d'entreprise réalisé sous l'angle de la pensée complexe. En effet, les auteurs ont conduit une recherche-action de quatre ans auprès d'un groupe du secteur de la santé qui souhaitait mobiliser la pensée morinienne afin d'aligner les pratiques et la stratégie sur les valeurs du groupe. Cette organisation associative d'un millier d'employés, accueillant 1500 personnes âgées réparties sur ses 24 établissements, avait en effet du mal à faire ancrer les valeurs de l'économie sociale et solidaire dans son fonctionnement. Le point singulier de cette recherche est que la pensée complexe n'était pas seulement le cadre d'investigation des chercheurs mais bel et bien l'approche qui séduisait les cadres praticiens porteurs du projet. La pensée complexe est donc un cadre épistémologique ou une façon de penser qui serait réservée aux chercheurs aguerris aux six tomes de La Méthode.

Dans un tout autre contexte, Audrey Missonier, Stéphanie Missonier, Frank Lasch et Anne-Sophie Thelisson se sont intéressés au cas des fusions d'entreprises. De telles opérations stratégiques, avec de tels enjeux financiers, économiques et organisationnels, sont souvent jalonnées de phases de doutes, de tensions et de conflits qui peuvent, au final, conduire à l'échec de l'intégration. L'étude de cas révèle comment une diversité de phénomènes dialogiques (engagements à long terme et à court terme, confiance et méfiance, autonomie et contrôle, cocréation et protection de connaissances) ont émergé lors du processus de fusion et comment ils se sont auto-alimentés au cours de phases d'ordre et de désordre. La pensée complexe offre une grille de lecture et de compréhension originale de ces paradoxes et l'article invite les managers à se dégager d'une perception négative du désordre et de l'instabilité dans les fusions, et à les considérer comme des phases inhérentes à une fusion et à la réinvention et la restabilisation du processus.

Pour sa part, Arnaud Stimec et Benoît Journé abordent le sujet sensible de la sûreté dans le secteur nucléaire. Alors que notre génération reste marquée par les catastrophes de Tchernobyl et de Fukushima, cet article vise à identifier les principes et pratiques de gouvernance qui contribuent à comprendre et faire face à la complexité de l'industrie nucléaire. À cet effet, l'auteur 
mobilise la pensée complexe pour se dégager de toute approche réductionniste et techno-centrée de ce qu'est la sûreté nucléaire et met en exergue l'importance des facteurs organisationnels et humains. L'étude du système de régulation de l'industrie nucléaire civile en France offre une interprétation originale d'une organisation tripartite dont, paradoxalement, l'instabilité tend à (ré)équilibrer le dialogue et favoriser le consensus.

Enfin, Bernard Fallery nous emporte vers un des problèmes éthiques majeurs soulevés par la digitalisation. Le projet Health data $h u b$, lancé en 2018 par le gouvernement, vise à la centralisation des données de santé publique pour servir des projets d'intelligence artificielle. L'objectif était de tirer avantage du potentiel des technologies numériques et de l'informatique afin de faciliter et d'améliorer les services de soin auprès des personnes. Toutefois, une pléthore de controverses a rapidement émergé du fait de la diversité des parties prenantes au projet (publiques ou privées). L'auteur dévoile les résultats d'une analyse de plus d'un millier de documents (courriels, compte rendus de réunions, rapports, enregistrements audios de débats, ordonnances de justice, articles de presse, etc.). La pensée complexe fait sens de ces controverses et permet à l'auteur de mettre en exergue trois boucles dialogiques, de poser la question éthique en termes de reliance et de proposer un modèle de gouvernance, non par délégation ou par régulation, mais par « auto-organisation ».

Comme vous le percevrez en les lisant, chacun de ces articles offre un éclairage sur la manière avec laquelle un problème complexe ne peut être simplement « posé » afin d'être résolu, mais doit davantage être « pensé » de manière évoluante lorsqu'il s'agit de le comprendre et de lui faire face. À la lumière de ces contributions, le message que je souhaite adresser à la communauté scientifique pour soutenir la pensée complexe dans la recherche sur les organisations, ne sera pas théorique ou épistémique, mais pratique. En effet, si l'œuvre d'Edgar Morin a été prolixe et a fait couler beaucoup d'encre, force est de constater qu'elle ne s'est pas traduite, du moins en France, par les changements paradigmatiques conséquents dans la manière de penser et de conduire la recherche. Les 1932 pages des 6 tomes de $L a$ Méthode $e^{3}$ n'y ont pas suffi, il n'était donc pas ici notre ambition de relever un même défi. Plus modestement, il nous semble pour autant pertinent de réfléchir sur la manière avec laquelle certains des « codes » attendus dans un article de recherche ou une thèse pourraient être repensés quant à la manière de les formuler.

Un point central de la pensée complexe sur les organisations réside dans la nécessaire analyse de leur caractère dynamique. Même si de toutes parts, des concepts de flexibilité, d'agilité, de transformation organisationnelle témoignent du principe « d'auto-éco-ré-organisation », les perspectives statiques et déterministes des théories institutionnelles et économiques de la première heure demeurent prégnantes, se font écho inconscient de la pensée cartésienne et des modélisations pseudomécanistes. Un premier point pour que les chercheurs puissent s'ouvrir davantage à la complexité de leur objet de recherche, est peut-être de revoir la manière avec laquelle

3. Pour les volumes poches des Éditions du Seuil. 
nous formulons une "problématique de recherche », censée fixée et devant être résolue par l'investigation méthodologique. Cette pratique académique centrale ne peut inconsciemment que contribuer à une rigidification de la représentation du phénomène étudié, même lorsque celui-ci est étudié de manière longitudinale. Dans les faits, combien de chercheurs n'ont jamais, en fonction des résultats de leurs observations du terrain, dû reformuler $a$ posteriori leurs hypothèses initiales afin de se conformer aux illusions de la logique déductive ? Aussi, si nous pouvions simplement inciter les chercheurs à concevoir leur recherche, non plus en termes de «problématique », mais de «problématisation », peut-être pourraient se dessiner les contours d'un nouveau cadre académique davantage favorable à l'analyse d'un objet de recherche à la lumière des phénomènes de désorganisation et réorganisation et des controverses qui lui sont inhérents. Dès lors, il ne serait plus question de considérer la problématique de recherche comme un point de départ ou un objectif à atteindre, mais comme un nécessaire processus évolutif. Dès lors, les auteurs auraient à revoir leurs questions de recherches ou hypothèses au fur et à mesure que leur investigation dévoilerait une évolution du problème étudié, et à délivrer aux lecteurs ce processus de requestionnement et de reformulation. Dès lors, il ne serait plus suffisant, pour le chercheur, de reconnaître sa non-neutralité, mais de conduire sa propre anthropologie et de dévoiler quels étaient ses croyances, intentions et états d'esprit liés aux différentes étapes du projet. Dès lors, il s'agirait de considérer la problématisation, non comme le «bon but final», mais comme une « conception sans but final» (Simon, 1969) où il s'agit davantage de modéliser « les processus cognitifs et affectifs par lesquels se sont formés, pas à pas, les "buts intermédiaires" auxquels se réfère l'élaboration raisonnée du prochain pas » (Le Moigne, 1977, p. XIV). Bien entendu, cette proposition n'a pas pour vocation de prétendre à la satisfaction du nécessaire changement de paradigme que requiert la pensée complexe. Elle vise tout au mieux, là encore, à proposer ce qui pourrait en être un premier pas en cherchant à questionner les pratiques académiques comme le dossier de la Revue française de gestion aura tenté de le faire. Pour autant, les meilleures intentions peuvent conduire aux pires résultats si à chacun de nos pas nous ne réinterrogeons pas le sens de nos actions. Passer de la «problématique » à la «problématisation »n'est pas un simple changement de vocabulaire, mais une transformation dans la manière de penser la recherche. Aussi, j'achèverai cet éditorial en remerciant chaleureusement mon ami Jean-Louis Le Moigne pour ses précieux conseils depuis plus de 20 ans et dont le dernier rappelait à ma mémoire ce texte, extrait de la quatrième de couverture du sixième tome de La Méthode, mettant si bien en exergue l'enjeu du « travailler à bien penser ».

« Si le devoir ne peut se déduire d'un savoir, le devoir a besoin d'un savoir.

La conscience morale ne peut se déduire de la conscience intellectuelle,

mais elle a besoin de la conscience intellectuelle, c'est-à-dire de pensée et de réflexion. En effet, la bonne intention risque de déterminer des actions mauvaises 
et la volonté morale d'avoir des conséquences immorales.

D'où la pertinence du précepte moral de Pascal :

"Travailler à bien penser".
Faire son devoir n'est souvent ni simple ni évident, mais incertain et aléatoire : c'est pourquoi l'éthique est complexe. » (Edgar Morin)

\section{BIBLIOGRAPHIE}

Avenier M.-J. (2019). "Les Sciences de l'artificiel : une conceptualisation révolutionnaire de sciences fondamentales à parachever", Projectique, vol. 3, n 19, p. 43-56.

Bouiss O. (2018). "Edgar Morin et la recherche en gestion », mémoire de recherche, université Paris-Dauphine.

Le Moigne J.-L. (1977). La théorie du système général, Presses universitaires de France.

Lévi-Strauss C. (1950). "Introduction à l'œuvre de Marcel Mauss", Sociologie et Anthropologie, Mauss M. (éd.), Presses universitaires de France, Paris.

Martinet A.-C. (2006). "Stratégie et pensée complexe", Revue française de gestion, vol. 32, $\mathrm{n}^{\mathrm{o}}$ 160, p. 31-46 (première édition 1993).

Morin E. (1977). La Méthode. Tome 1 : La nature de la nature, Éditions du Seuil, Paris.

Morin E. (1980). La Méthode. Tome 2 : La vie de la vie, Éditions du Seuil, Paris.

Morin E. (1986). La Méthode. Tome 3 : La connaissance de la connaissance, Éditions du Seuil, Paris.

Morin E. (1991). La Méthode. Tome 4 : Les idées, Éditions du Seuil, Paris.

Morin E. (2001). La Méthode. Tome 5 : L'humanité de l'humanité, Éditions du Seuil, Paris.

Morin E. (2004). La Méthode. Tome 6 : Éthique, Éditions du Seuil, Paris.

Morin E. et Le Moigne J.-L. (1999). L'intelligence de la complexité, L'Harmattan.

Rittel H.W.J. et Webber M.M. (1973). "Dilemmas in a general theory of planning", Policy Sciences, Springer, vol. 4, $\mathrm{n}^{\mathrm{o}}$ 2, p. 155-169.

Simon H.A. (1969). The Sciences of the Artificial, MIT Press, Cambridge, MA.

Simon H.A. (1995). "Problem forming, problem finding, and problem solving in design", Design and Systems: General Applications of Methodology, Collen A., Gasparski W.W. (Eds), p. 245-257. 